\title{
ADSORÇÃO DE BORO EM SOLOS ÁCRICOS EM FUNÇÃO DA VARIAÇÃO DO pH ${ }^{(1)}$
}

\author{
Marcio Roberto Soares ${ }^{(2)}$, José Carlos Casagrande ${ }^{(2)} \&$ Luis \\ Reynaldo Ferracciú Alleoni ${ }^{(3)}$
}

\begin{abstract}
RESUMO
A adsorção de B pelo solo é o principal fenômeno que afeta sua disponibilidade e seu potencial de contaminação. Os objetivos deste estudo foram: (a) investigar os efeitos do pH na adsorção de B em amostras superficiais e subsuperficiais de um Latossolo Vermelho Acriférrico (LVwf) e de um Latossolo Amarelo Ácrico (LAw) ambos com balanço positivo de cargas no horizonte Bw - e de um Nitossolo Vermelho eutroférrico (NVef); (b) avaliar a adequação do modelo de Langmuir em simular os resultados experimentais de adsorção; $\mathrm{e}$ (c) correlacionar os atributos químicos, físicos e mineralógicos dos solos com os valores de adsorção máxima $\left(\mathrm{Ads}_{\text {máx }}\right)$ e do coeficiente de afinidade $\left(\mathrm{K}_{\mathrm{L}}\right)$, derivados das isotermas. Experimentos do tipo "batch" foram realizados para determinação da quantidade de boro adsorvido, tendo como eletrólito-suporte soluções de $\mathrm{NaCl} 0,01 \mathrm{~mol} \mathrm{~L}^{-1} \operatorname{com} 0,1 ; 0,2$; 0,$4 ; 0,8 ; 1,2 ; 1,6 ; 2,0 ;$ e $4,0 \mu \mathrm{g} \mathrm{mL}^{-1}$ de $B$. Houve aumento da adsorção de $B$ com a elevação do $\mathrm{pH}$ e da concentração inicial de $\mathrm{B}$ adicionado. Maiores quantidades de $B$ foram adsorvidas na amostra superficial do NVef e nos horizontes Bw positivamente carregados dos Latossolos ácricos. A adsorção de $B$ foi representada por isotermas dos tipos C (linear) e $\mathrm{L}$ (exponencial) e adequadamente ajustada pelo modelo de Langmuir. Os parâmetros $A d s_{\text {máx }}$ e $\mathrm{K}_{\mathrm{L}}$ estimados por regressão não-linear não se correlacionaram com o pH. No pH natural, teores de matéria orgânica (MO) e de argila foram as variáveis que mais influenciaram a Ads $\mathrm{Aáx}_{\text {mas }}$ camadas superficiais. Nas camadas subsuperficiais, a Ads máx $_{\text {correlacionou-se }}$ negativamente com os teores de MO e positivamente com os de gibbsita. Teores de (hidr)óxidos de Fe cristalinos e amorfos estiveram correlacionados aos valores de $\mathrm{K}_{\mathrm{L}}$ obtidos nas amostras subsuperficiais e ao $\mathrm{pH}$ natural, bem como à Ads máx $_{\text {das }}$ camadas superficiais após aumento do $\mathrm{pH}$.
\end{abstract}

Termos de indexação: boro, adsorção, isotermas, Langmuir, ácrico.

\footnotetext{
(1) Recebido para publicação em novembro de 2006 e aprovado em outubro de 2007.

(2) Professor do Centro de Ciências Agrárias, Universidade Federal de São Carlos - UFSCar. Rodovia Anhanguera, km 174, Caixa Postal 153, CEP 13600-970 Araras (SP). E-mails: mrsoares@cca.ufscar.br; bighouse@power.ufscar.br

(3) Professor da Escola Superior de Agricultura "Luiz de Queiroz" - ESALQ/USP. Avenida Pádua Dias 11, Caixa Postal 09, CEP 13418-900 Piracicaba (SP). E-mail: lrfalleo@esalq.usp.br
} 


\title{
SUMMARY: BORONADSORPTION IN ACRIC SOILS AS A FUNCTION OF pH VARIATION
}

\begin{abstract}
Boron (B) adsorption by soils affects its bioavailable contents and contamination potential. The objectives of this study were: (a) to investigate the effects of $p H$ variation on $B$ adsorption by topsoil and subsoil samples from an Anionic "Rhodic" Acrudox (RA), an Anionic "Xanthic" Acrudox (XA) - both with a positive balance of charge in Bw horizon - and a Rhodic Kandiudalf (RK); (b) to evaluate the ability of the Langmuir model of simulating the experimental results of $B$ adsorption; and (c) to correlate chemical, physical and mineralogical soil attributes with the values of maximum adsorption (Ads $s_{\max }$ ) and affinity coefficient $\left(K_{L}\right)$, derived from the isotherms. To quantify the amount of adsorbed $B$, batch experiments were carried out using a $0.01 \mathrm{~mol} \mathrm{~L}^{-1} \mathrm{NaCl}$ solution as a support electrolyte containing $0.1 ; 0.2 ; 0.4 ; 0.8 ; 1.2 ; 1.6 ; 2.0$ and $4.0 \mu \mathrm{g} \mathrm{mL} L^{-1}$ of $B$. An increase of $B$ adsorption was observed after raising the $\mathrm{pH}$ (between 3.5 and 8.0) and the initial concentration of added $B$. Larger amounts of $B$ were adsorbed in topsoil samples from $R K$ and in $B W$ positively charged horizons from acric Oxisols. Boron adsorption was represented by type$C$ (linear) and type-L (exponential) isotherms and well fitted by the Langmuir model. $A d s_{\max }$ and $K_{L}$, estimated by nonlinear regressions, were not correlated with the $p H$. At a natural $\mathrm{pH}$, the organic matter $(O M)$ and clay contents influenced $A d s_{\max }$ most in the topsoil samples. In the subsoil layers, Ads $s_{\max }$ was negatively correlated with OM contents and positively correlated with gibbsite contents. At a natural $\mathrm{pH}$, the contents of free and amorphous $\mathrm{Fe}$ (hydr)oxides were correlated with $K_{L}$ values from subsoil samples. After $p H$ increase, the oxide contents were correlated with Ads $s_{\max }$ values from the topsoil samples.
\end{abstract}

Index terms: boron, adsorption, isotherms, tropical soils, Langmuir.

\section{INTRODUÇÃO}

A deficiência de boro (B) no solo constitui uma séria limitação para o desenvolvimento de diversas culturas de interesse econômico, em razão da baixa fertilidade natural de alguns solos, da maior remoção pelas colheitas e do uso crescente e continuado de fertilizantes e de corretivos, que contribuem para insolubilização de micronutrientes. Problemas de fitotoxicidade por B podem ocorrer por práticas inadequadas de fertilização, de irrigação e de aplicação de outros insumos agrícolas que contêm boro, como herbicidas e inseticidas (Keren \& Bingham, 1985). Atualmente, o potencial de entrada do B na cadeia alimentar, em concentrações que comprometem a saúde pública e a sanidade ambiental, aumentou com o descarte inadvertido de resíduos efluentes urbanos e industriais no solo. Assim, é importante conhecer o comportamento químico do boro no solo sob os pontos de vista agronômico e ambiental, cujo entendimento é complicado em razão da pequena amplitude entre suas concentrações no solo, que causam deficiência e toxicidade (Goldberg, 1997).

Geralmente, reações de adsorção governam a concentração de B na solução do solo e, portanto, definem a predisposição do elemento para absorção pelas plantas e o seu potencial de mobilidade no solo. (Hidr)óxidos de $\mathrm{Fe}, \mathrm{Al}$ e $\mathrm{Mn}$ adsorvem grande quantidade de B (Goldberg \& Glaubig, 1985; Keren \& Bingham, 1985; Goldberg et al., 1993), enquanto a caulinita é caracterizada pela baixa capacidade de adsorção de B (Keren \& Mezuman, 1981).
A distribuição do $\mathrm{B}$ entre as fases líquida e sólida é extremamente dependente do $\mathrm{pH}$, considerado o mais importante fator da solução do solo que afeta a disponibilidade de B. Geralmente, o B torna-se menos disponível com o aumento do $\mathrm{pH}$ da solução do solo. $\mathrm{O}$ $\mathrm{pH}$ atua tanto na definição da carga superficial dos colóides com interfaces variáveis quanto no controle da espécie predominante de $B$ na solução. Este elemento ocorre primariamente na forma não dissociada de ácido bórico $\left(\mathrm{H}_{3} \mathrm{BO}_{3}\right)$, na faixa de $\mathrm{pH}$ mais comum em solos tropicais. O predomínio de sua forma indissociada neutra se deve à sua alta constante de equilíbrio para reações de hidrólise $\left(\mathrm{p} K_{\mathrm{a}}=9,2\right)$ (Keren \& Bingham, 1985; Goldberg et al., 1993). Por essa razão, e dependendo da superfície reativa para adsorção, o boro pode ser facilmente lixiviado do solo.

A construção de isotermas de adsorção é um dos procedimentos mais utilizados no estudo da interação de ânions com o solo e é de grande utilidade para o pesquisador que procura uma relação numérica que descreva os resultados experimentais. A equação de Langmuir é atrativa por fornecer os coeficientes que quantificam a capacidade de adsorção máxima ( $\left.\mathrm{Ads}_{\operatorname{máx}}\right)$ e a energia de ligação $\left(\mathrm{K}_{\mathrm{L}}\right)$ e teve seu emprego bem sucedido na descrição da adsorção de B por (hidr)óxidos de $\mathrm{Fe}$ e de $\mathrm{Al}$ (Prodromou, 2004), por minerais de argila (Singh \& Mattigod, 1992), pela matéria orgânica (Gu \& Lowe, 1990; Arora et al., 2002) e por vários tipos de solo (Elrashidi \& O'Connor, 1982; Alleoni et al., 1998; Valladares et al., 1998; Alleoni \& Camargo, 2000; Azevedo et al., 2001; Chaudhary \& Shukla, 2004; Soares et al., 2005). 
Os propósitos deste estudo foram: investigar os efeitos do $\mathrm{pH}$ sobre a adsorção de $\mathrm{B}$ por solos altamente intemperizados; avaliar a habilidade da isoterma de Langmuir na descrição da adsorção de B por esses solos; e correlacionar atributos físicos, químicos e mineralógicos dos solos com os valores de máxima adsorção e da constante de afinidade obtidos do ajuste dos resultados experimentais pela isoterma de Langmuir.

\section{MATERIAL E MÉTODOS}

Foram estudadas amostras superficiais $(0-0,2 \mathrm{~m})$ e subsuperficiais (camada de maior expressão do horizonte B diagnóstico) de um Latossolo Vermelho Acriférrico (LVwf) argiloso e de um Latossolo Amarelo Ácrico (LAw) textura média - ambos com balanço positivo de cargas no horizonte $\mathrm{Bw}$ - e de um Nitossolo Vermelho eutroférrico (NVef), que serviu como referencial devido ao balanço negativo de cargas ao longo de todo o perfil. O LVwf e o NVef foram coletados em Ribeirão Preto (21 10 ' 39 ” S, $47^{\circ} 48$ ' 37 ” W), enquanto o LAw foi coletado em Guaíra (20॰19'06” S, 48 $18^{\circ} 18^{\prime} 38^{\prime}$ " W), ambos os municípios localizados no norte do Estado de São Paulo. Alguns atributos (eletro)químicos, físicos e mineralógicos dos solos encontram-se no quadro 1.

A caracterização química foi feita em amostras de terra fina seca ao ar (TFSA), passadas em peneira de malha de $2 \mathrm{~mm}$, conforme métodos descritos por Camargo et al. (1986) e apresentados por Soares et al. (2005). O pH foi potenciometricamente determinado em supensões de $\mathrm{H}_{2} \mathrm{O}$ e de $\mathrm{KCl} 1 \mathrm{~mol} \mathrm{~L}^{-1}$ com relação solo:solução 1:2,5, e a diferença $\Delta \mathrm{pH}=\mathrm{pH}_{\mathrm{KCl}}-\mathrm{pH}_{\mathrm{H}_{2} \mathrm{O}}$ foi usada como estimativa do balanço de cargas (Mekaru \& Uehara, 1972). O ponto de efeito salino nulo (PESN) representou o valor de $\mathrm{pH}$ no ponto de interseção das curvas de titulação com $\mathrm{H}^{+}$e com $\mathrm{OH}^{-}$, em três concentrações de $\mathrm{KCl}\left(0,1,0,01\right.$ e 0,001 $\mathrm{mol} \mathrm{L}^{-1}$ ) (Alleoni \& Camargo, 1994). Após saturação das amostras com $\mathrm{CsCl}$, a densidade de cargas foi calculada pela quantificação das cargas permanentes $\left(\sigma_{\mathrm{p}}\right)$ e variáveis $\left(\sigma_{\mathrm{v}}\right)$, com base na seletividade do Cs pelas cavidades siloxanas dos minerais de argila 2:1, que é maior do que pelas superfícies ionizáveis dos óxidos de Fe e de Al e das bordas quebradas dos minerais de argila 1:1 (Weber et al., 2005). A análise granulométrica foi feita pelo método da pipeta, ao passo que o método BET de adsorção de $\mathrm{N}_{2}$ foi empregado para medida da área superficial específica (ASE). A análise mineralógica qualitativa foi feita por difração de raios $\mathrm{X}$, e os teores de caulinita e de gibbsita foram semiquantificados por análise térmica diferencial (ATD).

Para estudo da adsorção de B em função da variação do $\mathrm{pH}$ do solo, subamostras foram incubadas até $\mathrm{pH}$ constante, após receberem doses crescentes de carbonato de cálcio e de ácido clorídrico para variar o pH de 4 até 8 (Casagrande \& Camargo, 1997). Experimentos de adsorção do tipo "batch" foram realizados para determinar a quantidade de boro adsorvido (Soares et al., 2005). A 2,0 g de TFSA foram adicionados $20 \mathrm{~mL}$ de solução de $\mathrm{NaCl} 0,01 \mathrm{~mol} \mathrm{~L}^{-1}$ contendo 0,$1 ; 0,2 ; 0,4 ; 0,8 ; 1,2 ; 1,6 ; 2,0 ;$ e $4,0 \mu \mathrm{g} \mathrm{mL}^{-1}$ de $\mathrm{B}$, na forma de ácido bórico. O conjunto solo-solução foi acondicionado em frascos de polietileno e colocado sob agitação $\left(150 \mathrm{osc} \mathrm{min}^{-1}\right)$ por $24 \mathrm{~h}$ a $24 \pm 2^{\circ} \mathrm{C}$. A suspensão foi centrifugada por 15 min a $2.000 \mathrm{rpm}$,

\section{Quadro 1. Alguns atributos (eletro)químicos, físicos e mineralógicos dos solos}

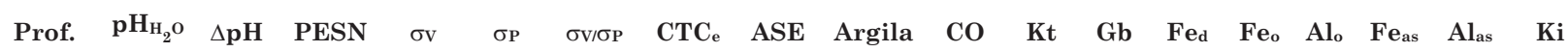

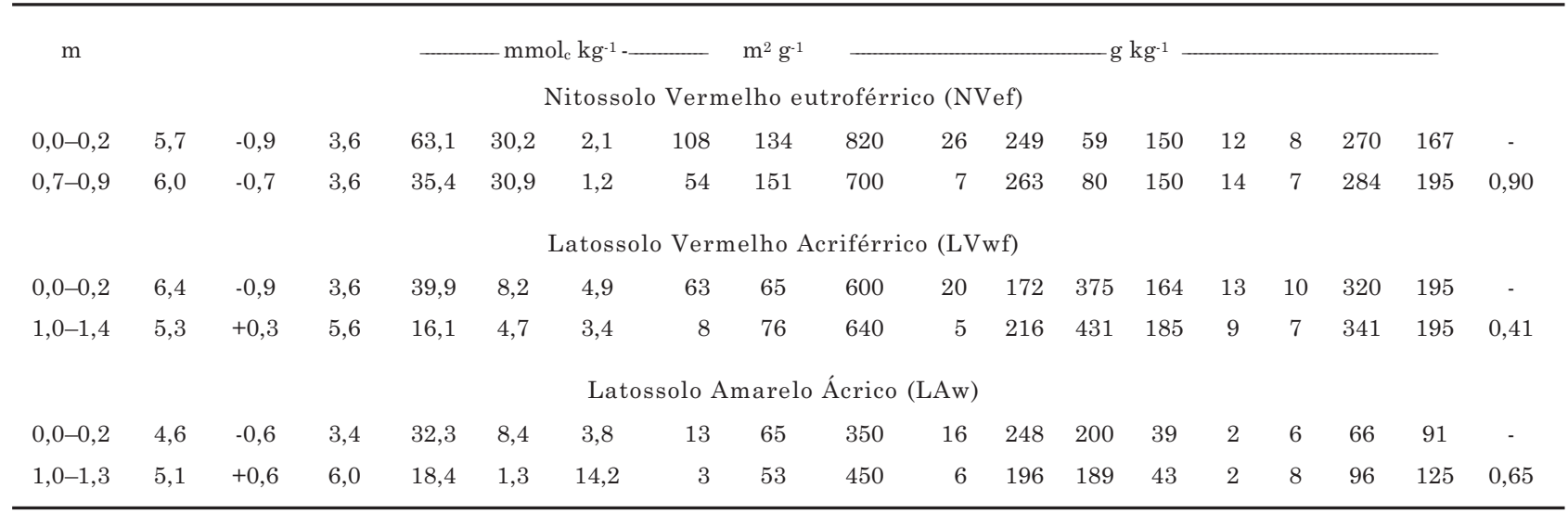

PESN: ponto de efeito salino nulo; $\sigma_{\mathrm{V}}$ : carga variável; $\sigma_{\mathrm{p}}$ : carga permanente; CTC : capacidade de troca de cátions efetiva; ASE:

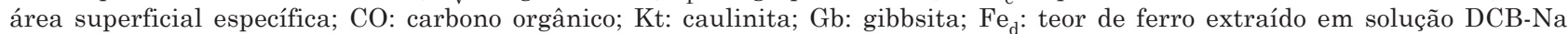
(ditionito-citrato-bicarbonato de sódio); $\mathrm{Fe}_{0}$ e $\mathrm{Al}_{\mathrm{o}}$ : teores de ferro e de alumínio extraídos em solução de Tamm $\left[(\mathrm{COOH})_{2} \cdot 2 \mathrm{H}_{2} \mathrm{O}+\left(\mathrm{NH}_{4}\right)_{2} \mathrm{C}_{2} \mathrm{O}_{4} \cdot \mathrm{H}_{2} \mathrm{O}\right] ; \mathrm{Fe}_{\text {as }}$ e $\mathrm{Al}_{\mathrm{as}}$ : teores de ferro e de alumínio extraídos pelo ataque com $\mathrm{H}_{2} \mathrm{SO}_{4} 9$ mol $\mathrm{L}^{-1}$; $\mathrm{Ki}$ índice de intemperização: $\mathrm{SiO}_{2} / \mathrm{Al}_{2} \mathrm{O}_{3}$. 
filtrada, e a concentração de boro remanescente no sobrenadante foi determinada pelo método colorimétrico da azometina-H. A quantidade de boro adsorvido ( $\mathrm{q}_{\mathrm{eq}}$ ) e a percentagem de adsorção (\%) foram calculadas pelas relações $\mathrm{q}_{\mathrm{eq}}=\left[\left(\mathrm{C}_{0}-\mathrm{C}_{\mathrm{eq}}\right) \mathrm{V}\right] / \mathrm{X} \mathrm{e} \% \mathrm{ads}=$ $\left[\left(\mathrm{C}_{0}-\mathrm{C}_{\mathrm{eq}}\right) / \mathrm{C}_{0}\right] \times 100$, respectivamente, em que $\mathrm{q}_{\mathrm{eq}}$ é a quantidade de boro adsorvido após o equilíbrio $\left(\mu \mathrm{g} \mathrm{g}^{-1}\right)$; $\mathrm{C}_{0}$ e $\mathrm{C}_{\text {eq }}$, a concentração inicial adicionada e a de equilíbrio $\left(\mu \mathrm{g} \mathrm{mL}^{-1}\right)$, respectivamente; $\mathrm{V}$, o volume de solução (mL); e X, a massa de amostra de terra (g). A quantidade de $\mathrm{B}$ originalmente presente nas amostras, apesar de pequena, foi descontada no cálculo da quantidade de boro adsorvido.

Isotermas de adsorção foram construídas a partir dos resultados experimentais, e a adsorção de B foi comparada com aquela estimada pela forma não-linear da isoterma de Langmuir, $\mathrm{q}_{\mathrm{eq}}=\left(\mathrm{K}_{\mathrm{L}} \mathrm{C}_{\mathrm{eq}} \mathrm{Ads}_{\text {máx }}\right) /$ $\left(1+\mathrm{K}_{\mathrm{L}} \mathrm{C}_{\mathrm{eq}}\right)$, em que $\mathrm{K}_{\mathrm{L}}$ é o parâmetro relacionado com

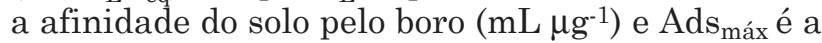
máxima capacidade de adsorção de $\mathrm{B}\left(\mu \mathrm{g} \mathrm{g}^{-1}\right)$. A isoterma de Langmuir foi ajustada aos resultados de adsorção de B pelos programas Fitfun.bas e Fitfunc (Barrow, 1987), os quais empregam a otimização nãolinear dos quadrados mínimos e não exigem a linearização da isoterma, o que evita tanto a introdução de mudanças na distribuição dos erros quanto a aquisição de parâmetros influenciados $\left(\mathrm{K}_{\mathrm{L}} \mathrm{e}\right.$ $\left.\mathrm{Ads}_{\text {máx }}\right)($ Goldberg et al., 1993).

$\mathrm{O}$ experimento foi realizado em delineamento inteiramente casualizado, em esquema fatorial $6 \times 4 \times 8$, sendo seis amostras de solos, quatro valores de $\mathrm{pH}$ e oito doses iniciais de $\mathrm{B}$. Os resultados foram a média aritmética de três repetições. Análises de correlação simples foram executadas para detectar os atributos químicos, físicos e mineralógicos que mais se correlacionaram com as constantes $\mathrm{K}_{\mathrm{L}}$ e $\mathrm{Ads}_{\text {máx }}$ de Langmuir e com a adsorção de $\mathrm{B}$ após adição de $2 \mathrm{mg} \mathrm{L}^{-1}$ de B (Alleoni \& Camargo, 2000). As análises estatísticas foram feitas com o auxílio do programa SAS (Statistical Analysis System) (SAS, 1988).

\section{RESULTADOS E DISCUSSÃO}

O modelo de Langmuir adequou-se bem aos valores de $\mathrm{B}$ adsorvido pelos solos, em toda a faixa de concentração e de $\mathrm{pH}$ estudada (Figura 1), por apresentar alto coeficiente de determinação $\left(\mathrm{R}^{2}=0,99^{* *}\right)$ e baixa soma dos quadrados dos desvios $(0,24<\mathrm{SQD}<3,54)$ entre os resultados experimentais e os estimados (Quadro 2) (Schultess \& Dey, 1996). Os altos coeficientes de determinação $\left(R^{2}=0,99^{*}\right)$ para o ajuste dos resultados experimentais à forma nãolinear da isoterma de Langmuir comprovaram a aderência dos resultados experimentais ao modelo (Quadro 2). Esses resultados eram esperados, porque não há registros de desvios da equação de Langmuir em concentrações inferiores a $30 \mu \mathrm{g} \mathrm{mL}^{-1}$ de B (Alleoni \& Camargo, 2000). Alleoni et al. (1998), investigando solos intemperizados brasileiros, utilizaram concentrações de B na solução que variaram de 0 a $16 \mathrm{mg} \mathrm{L}^{-1}$ e verificaram que os resultados de adsorção de $\mathrm{B}$ pelos solos foram bem ajustados pela isoterma de Langmuir. Outros autores também apontaram a habilidade da isoterma de Langmuir para reproduzir a adsorção de B por solos sob condições de baixa concentração (Elrashidi \& O'Connor, 1982; Alleoni \& Camargo, 2000; Soares et al., 2005).

Isotermas para o horizonte superficial do NVef e para o LVwf, principalmente em subsuperfície, exibiram formato tipo- $\mathrm{C}(\mathrm{C}=$ constante), sobretudo nos mais altos valores de $\mathrm{pH}$ (Figura 1). Nesse tipo de curva, o número e a energia dos sítios disponíveis para adsorção permanecem constantes ao longo de toda a amplitude de concentração e pode ocorrer a expansão da superfície disponível de maneira proporcional à quantidade adsorvida, até que todos os sítios de adsorção sejam ocupados. Isso pode estar relacionado com o surgimento de novas superfícies devido à precipitação de hidróxidos de alumínio após adição de $\mathrm{CaCO}_{3}$ (Alleoni \& Camargo, 2000). Todas as demais isotermas tiveram o típico formato tipo-L $(\mathrm{L}=$ Langmuir), mas não foram assintóticas, em razão das baixas concentrações $\left(\mathrm{C}_{0}\right)$. Dessa forma, a adsorção máxima $\left(\operatorname{Ads}_{\text {máx }}\right)$ não foi obtida diretamente da representação gráfica da isoterma, e sim calculada com o uso dos programas Fitfunc e Fitfun.bas (Barrow,1987). Nas curvas tipo-L há declínio da adsorção à medida que os sítios vão se tornando saturados. A partir da inclinação das curvas de adsorção, observou-se aumento na adsorção de B nas mais baixas concentrações. Provavelmente, com o aumento na concentração de B, mais sítios foram ocupados e a reação foi mais difícil de ocorrer, ocasionando diminuição da inclinação da curva (Figura 1).

Os valores das constantes de Langmuir (Quadro 2) foram semelhantes àqueles encontrados em estudos que utilizaram um intervalo semelhante para concentração de $\mathrm{B}$ adicionada aos solos. A constante

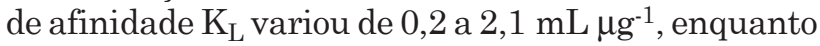
a adsorção máxima ( $\mathrm{Ads}_{\text {máx }}$ ) oscilou entre 32,5 e $128,6 \mu \mathrm{g} \mathrm{g}^{-1}$. A grande variação desses parâmetros foi atribuída às diferenças nos atributos químicos, físicos e mineralógicos das amostras. A comparação com resultados de outros autores é difícil, uma vez que as constantes estimadas pela equação de Langmuir variam conforme o intervalo de concentração utilizado na construção da isoterma. Para solos semelhantes, Alleoni et al. (1998) e Alleoni \& Camargo (2000) encontraram valores menores de $\mathrm{K}_{\mathrm{L}}\left(0,1-1,2 \mathrm{~mL} \mu \mathrm{g}^{-1}\right)$ e de $\operatorname{Ads}_{\text {máx }}\left(2,5-15,8 \mu \mathrm{g} \mathrm{g}^{-1}\right)$, que por sua vez foram muito próximos dos resultados reportados por Mezuman \& Keren (1981) para Alfissolos e Vertissolos, mas menores do que aqueles que Nicholaichuk et al. (1988) encontraram em diferentes solos agricultados do Canadá. Já para alguns solos de baixada do Rio de Janeiro e de Minas Gerais (Neossolo Aluvial, Gleissolo Háplico, Gleissolo Húmico e Organossolo), a Adssmáx 
Quadro 2. Parâmetros de ajuste da equação de Langmuir para resultados de adsorção de B por três solos intemperizados

\begin{tabular}{|c|c|c|c|c|c|c|c|c|}
\hline \multirow{2}{*}{$\begin{array}{c}\text { Profundidade } \\
\text { de } \\
\text { amostragem }\end{array}$} & \multirow[b]{2}{*}{$\mathbf{p H}$} & \multicolumn{3}{|c|}{ Constante de Langmuir } & \multicolumn{4}{|c|}{ Parâmetros da regressão } \\
\hline & & Adsmáx & $\mathbf{K}_{\mathrm{L}}$ & $* \mathrm{R}^{2}$ & SQD & $\begin{array}{c}\text { Intercepto } \\
(\alpha)\end{array}$ & $\begin{array}{l}\text { Coeficiente de } \\
\text { inclinação }(\beta)\end{array}$ & $* * \mathbf{R}^{2}$ \\
\hline \multirow[t]{2}{*}{$\mathrm{m}$} & & $\mu \mathrm{g} \mathrm{g}^{-1}$ & $\mathrm{~mL} \mu \mathrm{g}^{-1}$ & & & & & \\
\hline & \multicolumn{8}{|c|}{ Nitossolo Vermelho eutrófico (NVef) } \\
\hline \multirow[t]{4}{*}{$0,0-0,2$} & 4,2 & 95,1 & 0,22 & 0,99 & 0,51 & $-0,18$ & 1,01 & 0,99 \\
\hline & 5,2 & 81,7 & 0,31 & 0,99 & 0,73 & 0,60 & 0,96 & 0,99 \\
\hline & 5,9 & 76,5 & 0,49 & 0,99 & 0,99 & 0,92 & 0,95 & 0,99 \\
\hline & 7,7 & 119,5 & 0,29 & 0,99 & 1,33 & 1,23 & 0,95 & 0,99 \\
\hline \multirow[t]{5}{*}{$0,7-0,9$} & 3,5 & 61,2 & 1,03 & 0,99 & 1,26 & $-1,14$ & 1,07 & 0,99 \\
\hline & 3,9 & 32,5 & 1,25 & 0,99 & 2,61 & 2,48 & 0,91 & 0,96 \\
\hline & 5,9 & 41,3 & 0,68 & 0,99 & 0,57 & $-0,07$ & 1,00 & 0,99 \\
\hline & 7,8 & 33,6 & 2,13 & 0,99 & 0,97 & 1,16 & 0,94 & 0,99 \\
\hline & \multicolumn{8}{|c|}{ Latossolo Vermelho Acriférrico (LVwf) } \\
\hline \multirow[t]{4}{*}{$0,0-0,2$} & 4,8 & 58,9 & 0,22 & 0,99 & 0,34 & 0,41 & 0,95 & 0,99 \\
\hline & 5,6 & 48,3 & 0,31 & 0,99 & 0,54 & 0,21 & 0,98 & 0,99 \\
\hline & 7,9 & 65,4 & 0,49 & 0,99 & 1,05 & $-0,02$ & 1,00 & 0,99 \\
\hline & 7,9 & 120,4 & 0,29 & 0,99 & 1,04 & 0,73 & 0,97 & 0,99 \\
\hline \multirow[t]{5}{*}{$1,0-1,4$} & 3,8 & 48,8 & 1,03 & 0,99 & 0,58 & 0,30 & 0,98 & 0,99 \\
\hline & 5,4 & 78,5 & 1,25 & 0,99 & 0,49 & $-0,07$ & 1,01 & 0,99 \\
\hline & 7,1 & 123,4 & 0,68 & 0,99 & 0,67 & 0,36 & 0,97 & 0,99 \\
\hline & 8,0 & 128,6 & 2,13 & 0,99 & 0,24 & 0,70 & 0,96 & 0,99 \\
\hline & \multicolumn{8}{|c|}{ Latossolo Amarelo Ácrico (LAw) } \\
\hline \multirow[t]{4}{*}{$0,0-0,2$} & 3,7 & 50,7 & 0,51 & 0,99 & 0,92 & 0,86 & 0,94 & 0,99 \\
\hline & 5,0 & 37,3 & 0,32 & 0,99 & 0,66 & 0,50 & 0,95 & 0,99 \\
\hline & 6,0 & 47,4 & 0,30 & 0,99 & 0,48 & 0,36 & 0,97 & 0,99 \\
\hline & 7,9 & 36,1 & 1,21 & 0,95 & 3,54 & 4,02 & 0,85 & 0,96 \\
\hline \multirow[t]{4}{*}{$1,0-1,3$} & 3,7 & 34,6 & 0,50 & 0,99 & 0,56 & 0,42 & 0,97 & 0,99 \\
\hline & 4,4 & 44,7 & 0,46 & 0,99 & 0,81 & 0,75 & 0,95 & 0,99 \\
\hline & 4,9 & 62,2 & 0,39 & 0,99 & 0,36 & $-0,22$ & 1,00 & 0,99 \\
\hline & 6,1 & 50,4 & 0,64 & 0,99 & 0,49 & 0,25 & 0,99 & 0,99 \\
\hline
\end{tabular}

SQD: soma dos quadrados dos desvios; * $\mathrm{R}^{2}$ : coeficiente de determinação para ajuste à equação não-linear de Langmuir; $\alpha, \beta, \mathrm{e}$ $* * \mathrm{R}^{2}$ da equação linear $\mathrm{y}=\alpha+\beta \mathrm{x}$, em que y é a quantidade adsorvida observada e $\mathrm{x}$ é a quantidade adsorvida estimada pela isoterma de Langmuir.

foi de $6,1-10,3 \mu \mathrm{g} \mathrm{g}^{-1}$, no estudo de Valladares et al. (1998), e de 14,7 a 30,9 $\mu \mathrm{g} \mathrm{g}^{-1}$, no de Azevedo et al. (2001).

Os maiores valores de adsorção máxima estimados pelo modelo de Langmuir (em torno de $120 \mu \mathrm{g} \mathrm{g}^{-1}$ ) foram das amostras superficiais do NVef e LVwf e da subsuperficial do LVwf, sempre em $\mathrm{pH}$ acima de 7 (Quadro 2). O LAw apresentou os menores valores de $\mathrm{Ads}_{\text {máx, }}$ que estiveram próximos de $35 \mu \mathrm{g}$ g-1 e não apresentaram relação com o pH e com a profundidade de amostragem (Quadro 2). Nos horizontes superficiais, o B foi adsorvido mais fracamente, já que os valores de $\mathrm{K}_{\mathrm{L}}$ foram menores do que os das amostras subsuperficiais (Quadro 2), sugerindo a existência de reações de adsorção específica entre as espécies de $\mathrm{B}$ em solução e a superfície dos (hidr)óxidos de Fe e de $\mathrm{Al}$.

A adsorção de $\mathrm{B}$ foi muito dependente do $\mathrm{pH}$ da solução, tendo aumentado em função do pH no intervalo entre 3,5 e 8,0 (Figura 1). Vários estudos mostraram que a concentração de B na solução do solo tende a diminuir com o aumento do $\mathrm{pH}$ (Elrashidi \& O'Connor, 1982; Cruz et al., 1987; Goldberg et al., 1993). A adsorção de B aumenta no intervalo de $\mathrm{pH}$ 

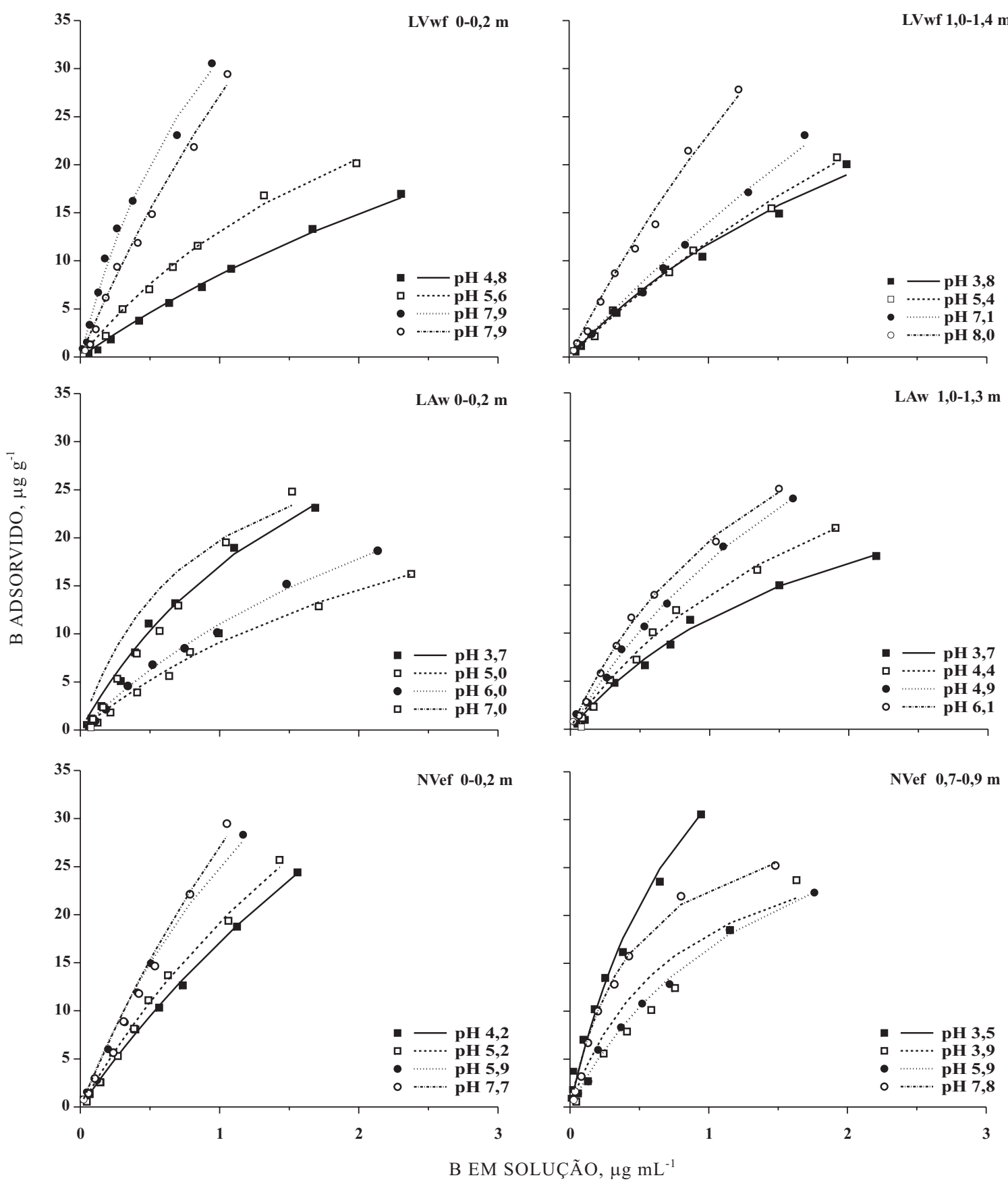

Figura 1. Isotermas de adsorção (curvas) obtidas a partir do ajuste da equação de Langmuir aos resultados experimentais (pontos). (LVwf = Latossolo Vermelho Acriférrico; LAw = Latossolo Amarelo Ácrico; NVef = Nitossolo Vermelho eutroférrico).

entre 3 e 9 e diminui na faixa de 10 a 11,5 , originando uma típica curva campaniforme com um pico de adsorção em torno de 9,0, muito próximo do $\mathrm{pK}_{\mathrm{a}}$ do ácido bórico (Keren \& Bingham, 1985; Goldberg et al., 2005). Evans (1987) atribuiu o aumento de adsorção de B ao aumento da proporção de ânions borato, que acompanha a elevação do $\mathrm{pH}$ e pode formar tanto complexos de esfera interna e externa com superfícies minerais, quanto complexos com a matéria orgânica. As duas espécies de $\mathrm{B}\left[\mathrm{B}(\mathrm{OH})_{4}{ }_{4}^{-} \mathrm{e} \mathrm{B}(\mathrm{OH})_{3}\right]$ possuem diferentes afinidades pelos colóides e aparecem em proporções variáveis na solução de equilíbrio em resposta a variações no $\mathrm{pH}$. Deve-se esperar, portanto, que a correção da acidez dos solos até a faixa de 5,5-6,0, como é recomendada para a maioria das culturas, aumente a adsorção do B naturalmente presente ou daquele adicionado via fertilização. 
Na camada de 0,7-0,9 m do NVef, a maior adsorção de $\mathrm{B}$ ocorreu a $\mathrm{pH} 3,5$. A amostra superficial do LAw adsorveu maior quantidade de $\mathrm{B}$ também nos valores mais baixos de $\mathrm{pH}(3,7$ e 5,0) (Figura 1). Sob condições muito ácidas, o $\mathrm{H}^{+}$é mais adsorvido do que $\mathrm{OH}^{-}$, e a superfície coloidal torna-se positivamente carregada, podendo ser balanceada por ânions da solução do solo, como o borato, por adsorção não-específica (Hingston et al., 1972).

Houve variação dos parâmetros de adsorção em decorrência das alterações no $\mathrm{pH}$ (Quadro 2), mas não houve distorção das estimativas de B adsorvido pela isoterma de Langmuir (Figura 1). Keren \& Bingham (1985) reportaram aumento da adsorção máxima com o incremento do $\mathrm{pH}$, o qual pode estar relacionado às mudanças na superfície dos colóides, favorecendo a formação de mais sítios de adsorção. A resposta da adsorção de $\mathrm{B}$ por argilominerais com respeito à variação do $\mathrm{pH}$ pode ser explicada da seguinte maneira (Keren \& Bingham,1985): abaixo do $\mathrm{pH}$ 7,0, a espécie $\mathrm{B}(\mathrm{OH})_{3}$ predomina e, uma vez que a afinidade dos argilominerais pelo ácido bórico é baixa, a quantidade de $\mathrm{B}$ adsorvido tende a ser pequena. Na faixa ácida de $\mathrm{pH}$, as concentrações de $\mathrm{B}(\mathrm{OH})_{4}{ }^{-}$e de $\mathrm{OH}^{-}$são baixas, e sua contribuição ao total de B adsorvido é pequena, mesmo com maior afinidade pelos minerais de argila. Com aumento do $\mathrm{pH}$ até próximo de 9,0 , a proporção de $\mathrm{B}(\mathrm{OH})_{4}{ }^{-}$aumenta rapidamente, porém a concentração de $\mathrm{OH}^{-}$é ainda pequena para exercer efetiva competição com ânions borato. A partir desse valor, seguidos aumentos de $\mathrm{pH}$ resultam no aumento da concentração de $\mathrm{OH}^{-}$em relação à de $\mathrm{B}(\mathrm{OH})_{4}{ }^{-}$, e a adsorção de $\mathrm{B}$ decresce devido à competição com $\mathrm{OH}^{-}$ pelos sítios de adsorção. Em $\mathrm{pH}$ abaixo de 7,0, o ácido bórico está predominantemente indissociado, mas a pH 9,2 está 50\% dissociado. A proporção de ácido bórico e de ânions tetraidroxiborato a pH 7,0 é, respectivamente, 99 e 1\%, aumentando para 80 e $18 \%$ quando o $\mathrm{pH}$ sobe para 8,4 (Keren \& Bingham, 1985). Dessa forma, é razoável admitir que a adsorção de B na forma de ânions tenha sido dificultada nas amostras do LVwf e do LAw, cujos valores de $\mathrm{pH}$ sob condições naturais variaram de 4,8 a 5,4 , e do NVef, onde o $\mathrm{pH}$ era de 5,9 nas duas amostras. $\mathrm{O}$ uso de $\mathrm{NaCl}$ como eletrólito-suporte favorece a dissociação do ácido bórico para produção de ânions borato a um determinado $\mathrm{pH}$ (Keren \& Bingham, 1985). Portanto, pode-se considerar esse efeito mesmo que a concentração de $\mathrm{NaCl}$ utilizada tenha sido baixa $\left(0,01 \mathrm{~mol} \mathrm{~L}^{-1}\right)$.

Não houve correlação significativa entre o $\mathrm{pH}$ das amostras superficiais sob condições naturais (testemunha) e os parâmetros de adsorção derivados da isoterma de Langmuir ( $\mathrm{Ads}_{\text {máx }}$ e $\mathrm{K}_{\mathrm{L}}$ ) (Quadro 3). A falta de correlação entre a adsorção máxima e o pH foi reportada anteriormente por Azevedo et al. (2001) em solos de várzea de Minas Gerais. Por outro lado, a

Quadro 3. Coeficientes de correlação da análise de regressão simples entre os parâmetros de adsorção das isotermas de Langmuir ( $\mathrm{Ads}_{\text {máx }}$ e $\mathrm{K}_{\mathrm{L}}$ ) e de adsorção após adição de $2 \mathrm{mg} \mathrm{L}^{-1}$ de $\mathrm{B}$ e alguns atributos dos solos

\begin{tabular}{|c|c|c|c|c|c|c|c|c|c|c|c|c|c|c|c|}
\hline \multicolumn{2}{|c|}{$\begin{array}{l}\text { Coeficiente de adsorção } \\
\text { de Langmuir }\end{array}$} & $\mathrm{pH}$ & $\sigma_{P}$ & $\sigma \mathrm{v}$ & CTC $_{e}$ & Argila & $\mathrm{CO}$ & ASE & $\mathrm{Fe}_{\text {as }}$ & $\mathrm{Fe}_{\mathrm{d}}$ & $\mathrm{Fe}_{\mathrm{o}}$ & $\mathrm{Al}_{\text {as }}$ & $\mathrm{Al}_{\mathrm{o}}$ & $\mathbf{K t}$ & Gb \\
\hline \multirow{3}{*}{$\begin{array}{l}\text { Adsmáx ao } \mathrm{pH} \\
\text { natural }\end{array}$} & $\begin{array}{l}\text { Todas as } \\
\text { amostras }\end{array}$ & 0,16 & 0,08 & $-0,10$ & 0,20 & 0,53 & 0,12 & $-0,04$ & 0,50 & 0,50 & 0,20 & 0,41 & 0,35 & $-0,80$ & $0,99^{*}$ \\
\hline & Superfície & 0,65 & 0,95 & 0,83 & $0,99^{*}$ & $0,99 * * *$ & $0,99^{*}$ & 0,84 & 0,80 & 0,84 & 0,85 & 0,75 & 0,55 & - & - \\
\hline & Subsuperfície & $-0,79$ & $-0,94$ & $-0,85$ & $-0,85$ & $-0,30$ & $-0,99 * *$ & $-0,78$ & 0,15 & 0,167 & $-0,48$ & $-0,07$ & 0,02 & $-0,80$ & $0,99^{*}$ \\
\hline \multirow{3}{*}{$\begin{array}{l}\text { Adsmáx ao } \mathrm{pH} \\
\text { mais alto }\end{array}$} & Todas as & 0,42 & 0,25 & $-0,07$ & 0,37 & 0,55 & 0,38 & $-0,06$ & 0,71 & $0,72^{*}$ & 0,46 & 0,58 & 0,54 & $-0,48$ & 0,83 \\
\hline & $\begin{array}{l}\text { amostras } \\
\text { Superfície }\end{array}$ & 0,93 & 0,68 & 0,49 & 0,80 & 0,88 & 0,80 & 0,49 & 0,98 & $0,99 * *$ & $0,99 * *$ & 0,97 & 0,87 & - & - \\
\hline & Subsuperfície & $-0,46$ & $-0,72$ & $-0,55$ & $-0,55$ & 0,12 & $-0,94$ & $-0,45$ & 0,55 & 0,56 & $-0,07$ & 0,35 & $-0,54$ & 0,83 & $-0,93$ \\
\hline \multirow{3}{*}{$\begin{array}{l}\mathrm{K}_{\mathrm{L}} \text { ao } \mathrm{pH} \\
\text { natural }\end{array}$} & $\begin{array}{l}\text { Todas as } \\
\text { amostras }\end{array}$ & $-0,09$ & $-0,41$ & $-0,01$ & $-0,29$ & 0,35 & $-0,56$ & 0,20 & 0,51 & 0,54 & 0,19 & 0,47 & $-0,40$ & 0,01 & 0,46 \\
\hline & Superfície & $-0,25$ & 0,82 & 0,93 & 0,70 & 0,59 & 0,71 & 0,93 & $-0,03$ & 0,05 & 0,07 & $-0,11$ & $-0,37$ & - & - \\
\hline & Subsuperfície & 0,03 & $-0,29$ & $-0,08$ & $-0,07$ & 0,59 & $-0,65$ & 0,04 & 0,89 & $0,99 * *$ & $0,99 * *$ & 0,76 & $-0,52$ & 0,01 & 0,46 \\
\hline \multirow{3}{*}{$\begin{array}{l}\mathrm{K}_{\mathrm{L}} \text { ao } \mathrm{pH} \\
\text { mais alto }\end{array}$} & $\begin{array}{l}\text { Todas as } \\
\text { amostras }\end{array}$ & $-0,18$ & $-0,53$ & 0,08 & $-0,46$ & 0,01 & $-0,73^{*}$ & 0,27 & 0,21 & 0,24 & 0,08 & 0,25 & $-0,66$ & 0,66 & $-0,24$ \\
\hline & Superfície & $-0,92$ & $-0,69$ & $-0,49$ & $-0,80$ & $-0,88$ & $-0,80$ & $-0,50$ & $-0,98$ & 0,97 & 0,91 & $-0,97$ & $-0,87$ & - & - \\
\hline & Subsuperfície & 0,67 & 0,40 & 0,59 & 0,59 & 0,97 & 0,00 & 0,68 & 0,98 & 0,89 & 0,42 & 0,95 & 0,12 & 0,66 & $-0,24$ \\
\hline \multirow{3}{*}{$\begin{array}{l}\text { Adsorção após } \\
\text { adição de } \\
2 \mu \mathrm{g} \mathrm{mL}^{-1} \mathrm{~B}\end{array}$} & $\begin{array}{l}\text { Todas as } \\
\text { amostras }\end{array}$ & $-0,02$ & 0,39 & 0,59 & 0,43 & 0,55 & 0,08 & 0,61 & $-0,03$ & $-0,01$ & 0,12 & 0,02 & $-0,14$ & 0,19 & $-0,62$ \\
\hline & Superfície & $-0,02$ & 0,92 & $0,99^{*}$ & 0,85 & 0,76 & 0,85 & $0,99^{*}$ & 0,18 & 0,27 & 0,29 & 0,11 & $-0,15$ & - & - \\
\hline & Subsuperfície & 0,17 & 0,47 & 0,28 & 0,27 & $-0,42$ & 0,79 & 0,16 & $-0,78$ & $-0,79$ & $-0,23$ & $-0,62$ & 0,02 & 0,19 & $-0,62$ \\
\hline
\end{tabular}

$\sigma_{\mathrm{P}}$ : cargas elétricas permanentes; $\sigma_{\mathrm{V}}$ : cargas elétricas variáveis; $\mathrm{CTC}_{\mathrm{e}}$ : capacidade de troca de cátions efetiva; CO: carbono orgânico; ASE: área superficial específica; $\mathrm{Fe}_{\mathrm{as}}$ e $\mathrm{Al}_{\mathrm{as}}$ : ferro e alumínio extraídos por ataque sulfúrico; $\mathrm{Fe}_{\mathrm{d}}$ : teor de ferro cristalino extraído por solução de ditionito-citrato bicarbonato de sódio; $\mathrm{Fe}_{\mathrm{o}}$ e $\mathrm{Al}_{\mathrm{o}}$ : teores de $\mathrm{Fe}$ e de $\mathrm{Al}$ pouco cristalinos extraídos por solução ácida de oxalato de amônio; Kt: caulinita; Gb: gibbsita; *, **, *** significativo a 10, 5 e $1 \%$, respectivamente. 
$\mathrm{Ads}_{\text {máx }}$ esteve correlacionada com o pH de 20 solos canadenses, pertencentes a diferentes classes (Evans, 1987).

A adsorção máxima ( $\left.\mathrm{Ads}_{\operatorname{máx}}\right)$ estimada pelo modelo de Langmuir no $\mathrm{pH}$ natural das amostras apresentou o maior número de correlações significativas com os atributos dos solos (Quadro 3). Alleoni \& Camargo (2000) não encontraram correlações significativas entre as constantes de Langmuir e os atributos químicos, como o pH, os teores de matéria orgânica e a CTC de um Latossolo Vermelho distrófico, de três Latossolos álicos e de um Argissolo eutrófico. Já Elrashidi \& O'Connor (1982) e Chaudhary \& Shukla (2004) incluíram os teores de matéria orgânica e de argila e a CTC entre as variáveis que mais influenciaram a adsorção de B por Aridissolos, Inceptissolos e Alfissolos.

Um alto $(r=0,99)$ e significativo $(p<0,01)$ coeficiente de correlação foi obtido para a relação entre os teores de argila das amostras superficiais e a $\mathrm{Ads}_{\text {máx }}$ (Quadro 3). Evans (1987) e Nicholaichuk et al. (1988), estudando solos canadenses pouco intemperizados, mostraram aumento da adsorção máxima de B com o aumento da proporção de argila. Alleoni \& Camargo (2000) não só encontraram correlação entre o teor de argila e a adsorção de $\mathrm{B}\left(\mathrm{r}=0,79^{* *}\right)$, como também observaram que $62 \%$ da variação do B adsorvido foi explicada pelos teores de argila na análise de regressão múltipla.

Keren \& Mezuman (1981) avaliaram a adsorção de $\mathrm{B}$ por diferentes minerais de argila, como a ilita, a montmorilonita e a caulinita, e observaram que os três argilominerais adsorveram maior quantidade de $\mathrm{B}$ com o aumento do $\mathrm{pH}$ da solução. Nas amostras deste estudo, a caulinita foi um importante constituinte mineralógico dos solos (Quadro 1). Azevedo et al. (2001) encontraram correlação negativa entre os teores de caulinita e a adsorção máxima $\left(\mathrm{r}=-0,71^{*}\right)$, indicando baixa reatividade do mineral para adsorção de B em solos de baixada. A correlação entre a adsorção máxima e os teores de caulinita também foi negativa, mas não-significativa (Quadro 3).

A composição mineralógica semelhante do LVwf, do LAw e do NVef, com predomínio de gibbsita e de caulinita na fração argila desferrificada e altos teores de (hidr)óxidos de $\mathrm{Fe}$ e de $\mathrm{Al}$ cristalinos e amorfos (Quadro 1), favoreceu a correlação positiva e significativa da fração argila para adsorção de B (Quadro 3). Estes minerais formam complexos do tipo esfera interna (inner-sphere) com o B, o que reforça a hipótese de ocorrência de adsorção específica deste elemento (Goldberg et al., 1993).

$\mathrm{Em} \mathrm{pH}$ natural, teores de gibbsita das amostras subsuperficiais apresentaram correlação positiva com a Ads máx de B, enquanto os teores de (hidr)óxidos de Fe amorfos e cristalinos não se correlacionaram com a $\mathrm{Ads}_{\text {máx }}$. Coeficientes de correlação mais altos são esperados com (hidr)óxidos de $\mathrm{Al}$ do que com os de $\mathrm{Fe}$, devido à alta afinidade do $\mathrm{B}$ pelos grupos $\mathrm{OH}$ dos (hidr)óxidos de $\mathrm{Al}$ e à maior superfície específica (Sims $\&$ Bingham, 1968). Espera-se mesmo comportamento para (hidr)óxidos amorfos, apesar de o efeito combinado da MO com a fração argila poder mascará-lo (Goldberg \& Glaubig, 1985). Entretanto, esse argumento foi refutado com os resultados obtidos com a $\mathrm{Ads}_{\text {máx }}$ medida no mais alto valor de $\mathrm{pH}$ do solo (Quadro 3). Tanto os (hidr)óxidos de Fe amorfos como os cristalinos estiveram fortemente correlacionados com a adsorção máxima, como observado anteriormente por $\mathrm{Gu} \&$ Lowe (1990). Quanto maior o teor de (hidr)óxidos de Fe livres, maior é a adsorção máxima (Mezuman \& Keren, 1981). Sims \& Bingham (1968) e Goldberg \& Glaubig (1985) afirmam que a capacidade de adsorção de B por um solo aumenta proporcionalmente aos seus teores de (hidr)óxidos pouco cristalinos. A Ads máx estimada para as amostras dos horizontes subsuperficiais dos dois Latossolos ácricos pode estar associada com o alto valor do PESN, que, por ser maior do que o $\mathrm{pH}$ do solo, favoreceu o predomínio de cargas positivas, as quais podem atuar na adsorção eletrostática dos poucos ânions borato existentes em condições em que o pH do solo está distante da primeira constante de dissociação do ácido bórico (Soares et al., 2005).

Um alto, positivo e significativo coeficiente de correlação $\left(r=0,99^{*}\right)$ foi obtido entre os teores de matéria orgânica e a máxima adsorção pelas amostras superficiais (Quadro 3). A matéria orgânica do solo adsorve mais $\mathrm{B}$ do que os constituintes minerais ( $\mathrm{Gu}$ \& Lowe, 1990; Yermiyahu et al., 1995). Segundo Azevedo et al. (2001), a forte correlação entre o teor de matéria orgânica e a $\operatorname{Ads}_{\text {máx }}\left(\mathrm{r}=0,82^{* *}\right)$ foi indicativo de que os sítios mais ativos para adsorção de B pertencem aos colóides orgânicos. Valladares et al. (1998) obtiveram teores mais elevados de B adsorvido em amostras superficiais de solos de baixada, o que foi atribuído à capacidade de 0 ânion borato ligar-se aos compostos orgânicos, favorecendo o mecanismo de adsorção pela formação de complexos do tipo boratidol. $\mathrm{O}$ efeito da matéria orgânica é mais pronunciado quando seus teores no solo são maiores do que $15 \mathrm{mg} \mathrm{dm}^{-3}$ (Mezuman \& Keren,1981), como é o caso do NVef, do LVwf e do LAw (Quadro 1).

Alguns autores obtiveram conclusões controvertidas com respeito ao efeito da matéria orgânica sobre a adsorção de B. Alleoni \& Camargo (2000) não encontraram associação entre os teores de $\mathrm{B}$ adsorvido e a matéria orgânica do solo. Mezuman \& Keren (1981) e Arora et al. (2002) notaram diminuição da adsorção de boro por Aridissolos com a remoção da matéria orgânica. Por outro lado, a remoção da matéria orgânica aumentou a capacidade de adsorção de B por Inceptissolos estudados por Marzadori et al. (1991). Em 20 solos canadenses com diferentes atributos, Evans (1987) encontrou forte correlação entre $A d s_{\text {máx }}$ e a matéria orgânica, mas somente com $\mathrm{pH}$ acima de 7,0. No presente estudo, os teores de matéria orgânica e os valores de adsorção máxima no mais alto $\mathrm{pH}$ não estiveram correlacionados (Quadro 3). 
Em solos ou horizontes em que a matéria orgânica desempenha importante função na adsorção de B, a calagem pode fornecer uma concentração de Ca capaz de saturar sítios de adsorção representados pelos grupos carboxílicos dos ácidos húmicos, diminuindo a quantidade de sítios disponíveis para adsorção de B (Gu \& Lowe, 1990). O aumento do pH não implica necessariamente redução da disponibilidade de B nas camadas superficiais, já que a calagem estimula a mineralização da matéria orgânica, principal fonte de B no solo (Cruz et al., 1987). Há, porém, a possibilidade de formação de complexos entre ácidos orgânicos dissolvidos e o B, o que pode acentuar a adsorção (Prodromou, 2004).

Em subsuperfície, os teores de matéria orgânica estiveram inversamente correlacionados com a máxima adsorção de B (Quadro 3). O aumento da adsorção com o decréscimo dos teores de matéria orgânica se deve à ativação dos sítios de adsorção da superfície dos (hidr)óxidos de Fe e de Al. Alguns sítios de adsorção estão presentes somente nas superfícies minerais e não operam se estiverem obstruídos pela matéria orgânica (Marzadori et al., 1991).

A CTC apresentou correlação positiva com a adsorção de B $\left(r=0,99^{*}\right)$ (Quadro 3), mas é considerada uma variável que depende dos conteúdos de matéria orgânica e de argila, já que a CTC teoricamente não pode contribuir para adsorção de espécies negativamente carregadas. Esse argumento foi confirmado nas amostras subsuperficiais, quando a CTC apresentou correlação negativa e não-significativa $(r=-0,94)$.

Após adição de $2 \mathrm{mg} \mathrm{L}^{-1}$ de $\mathrm{B}$, a adsorção de $\mathrm{B}$ dependeu da quantidade de cargas variáveis $\left(\sigma_{\mathrm{V}}\right)$ e da área superfícial específica (ASE) (Quadro 3). A maior área de exposição da superfície às espécies de $\mathrm{B}$ da solução do solo, associada ao maior número de grupos hidroxilados para troca de ligantes com o B, favorece a maior retenção do elemento. $\mathrm{O}$ valor de $\mathrm{r}$ para a variável ASE $(r=0,99 *)$ foi superior ao encontrado por Alleoni \& Camargo (2000). A superfície específica também foi variável importante na adsorção de B pelos solos de baixada estudados por Azevedo et al. (2001).

$\mathrm{O}$ coeficiente de afinidade $\left(\mathrm{K}_{\mathrm{L}}\right)$ pode oferecer uma percepção sobre o provável mecanismo envolvido na adsorção de $\mathrm{B}$. Os valores de $\mathrm{K}_{\mathrm{L}}$, estimados para as amostras no $\mathrm{pH}$ natural, apresentaram correlação estatisticamente significativa com os conteúdos de (hidr)óxidos de Fe cristalinos $(r=0,99 * *)$ e amorfos $(r=0,99 * *)$ (Quadro 3). Valores de $\mathrm{K}_{\mathrm{L}}$ obtidos no mais alto valor de $\mathrm{pH}$ indicaram que a matéria orgânica correlacionou-se negativamente $\left(r=-0,73^{*}\right)$ com a afinidade do B pelo solo, independentemente da profundidade (Quadro 3). Embora a matéria orgânica favoreça a adsorção de maior quantidade de boro, as constantes $\mathrm{K}_{\mathrm{L}}$ estimadas pelo modelo de Langmuir sugeriram que o elemento foi mais fortemente adsorvido nos horizontes subsuperficiais, onde a matéria orgânica teve menor influência. Em subsuperfície, provavelmente o
B foi adsorvido por mecanismos de esfera interna, com alta energia de ligação, como indicado pelos altos valores de $\mathrm{K}_{\mathrm{L}}$ (Quadro 2). Esse mecanismo de complexação inner-sphere é de ocorrência predominante em superfícies com cargas reversíveis, típica de solos extremamente intemperizados. Ânions borato tornamse especificamente adsorvidos pelo mecanismo de troca de ligantes, pelo qual compostos de B deslocam grupos hidroxilados reativos da superfície para formação de ligações covalentes de grande estabilidade (Goldberg et al., 1993). Além da formação de complexos de esfera interna (inner-sphere), sua forma aniônica $\left[\mathrm{B}(\mathrm{OH})_{4}^{-}\right]$ também pode ser retida por meio de atração eletrostática, pela formação de complexos de esfera externa (Goldberg, 1997). Como a matéria orgânica oferece sítios de menor energia, os mais baixos valores de $K_{L}$ sugeriram o predomínio de adsorção eletrostática. Possivelmente, o pH não favoreceu a ocorrência de grandes proporções de ânions borato, em razão da elevada constante de dissociação do ácido bórico. No entanto, a adsorção específica de boro, que ocorre independentemente do sinal da carga líquida superficial dos colóides, pode ocorrer com as espécies $\mathrm{B}(\mathrm{OH})_{3}$ e $\mathrm{B}(\mathrm{OH})_{4}^{-}($Keren \& Bingham, 1985).

\section{CONCLUSÕES}

1. Houve incremento da adsorção de B com o aumento do $\mathrm{pH}$.

2. A adsorção aumentou com o incremento da concentração de B.

3. A isoterma de Langmuir adequou-se bem aos valores de $\mathrm{B}$ adsorvido pelos solos, em toda a faixa de concentração e de pH, e mostrou-se um modelo válido para determinar os parâmetros relacionados à capacidade de máxima adsorção e à afinidade de adsorção.

4. Os maiores valores de adsorção máxima estimados pelo modelo de Langmuir foram das amostras superficiais do NVef e do LVwf e da subsuperficial do LVwf, sempre em $\mathrm{pH}$ acima de 7,0.

5. A maior afinidade para adsorção de B foi quantitativamente mostrada pelos maiores valores de coeficiente de afinidade das amostras subsuperficiais.

\section{AGRADECIMENTO}

À FAPESP, pela concessão de bolsa ao primeiro autor (processo n. 98/02386-1).

\section{LITERATURA CITADA}

ALLEONI, L.R.F. \& CAMARGO, O.A. Boron adsorption in soils from the State of São Paulo, Brazil. Pesq. Agropec. Bras., 35:413-421, 2000. 
ALLEONI, L.R.F. \& CAMARGO, O.A. Pontos de efeito salino nulo de Latossolos ácricos. R. Bras. Ci. Solo, 18:175-180, 1994.

ALLEONI, L.R.F.; CAMARGO, O.A. \& CASAGRANDE, J.C. Isotermas de Langmuir e de Freundlich na descrição da adsorção de boro em solos altamente intemperizados. Sci. Agric., 55:379-387, 1998.

ARORA, H.; BHARDWAJ, S.S. \& SHARMA, B.D. Effect of organic matter on boron adsorption by some soils of Punjab. Asian J. Chem., 14:746-752, 2002.

AZEVEDO, W.R.; FAQUIN, V. \& FERNANDES, L.A. Adsorção de boro em solos de várzea do Sul de Minas Gerais. Pesq. Agropec. Bras., 36:957-964, 2001.

BARROW, N.J. Reactions with variable-charge soils. Dordrecht, Martinus Nijhoff Publishers, 1987. 191p.

CAMARGO, O.A.; MONIZ A.C.; JORGE, J.A. \& VALADARES, J.M.A.S. Métodos de análise química, mineralógica e física de solos do IAC. Campinas, Instituto Agronômico de Campinas, 1986. 94p. (Boletim Técnico, 106)

CASAGRANDE, J.C. \& CAMARGO, O.A. Adsorção de fosfato em solos com caráter ácrico avaliada por um modelo de complexação de superfície. R. Bras. Ci. Solo, 21:353-360, 1997.

CHAUDHARY, D.R. \& SHUKLA, L.M. Boron adsorption and desorption in arid soils of India. Agrochimica, 48:141-152, 2004.

CRUZ, M.C.P.; NAKAMURA, A.M. \& FERREIRA, M.E. Adsorção de boro pelo solo: Efeito da concentração e do pH. Pesq. Agropec. Bras., 22:621-626, 1987.

ELRASHIDI, M.A. \& O'CONNOR, G.A. Boron sorption and desorption in soils. Soil Sci. Soc. Am. J., 46:27-31, 1982.

EVANS, L.J. Retention of boron by agricultural soils from Ontario. Can. J. Soil Sci., 67:33-42, 1987.

GOLDBERG, S. \& GLAUBIG, R.A. Boron adsorption on aluminum and iron oxide minerals. Soil Sci. Soc. Am. J., 49:1374-1379, 1985

GOLDBERG, S. Reactions of boron with soils. Plant Soil, 193:35-48, 1997.

GOLDBERG, S.; CORWIN, D.L.; SHOUSE, P.J. \& SUAREZ, D.L. Prediction of boron adsorption by field samples of diverse textures. Soil Sci. Soc. Am. J., 69:1379-1388, 2005.

GOLDBERG, S.; FORSTER, H.S. \& HEICK, E.L. Boron adsorption mechanisms on oxides, clay minerals and soils inferred from ionic strength effects. Soil Sci. Soc. Am. J., 57:704-708, 1993.

GU, B. \& LOWE, L.E. Studies on the adsorption of boron on humic acids. Can. J. Soil Sci., 70:305-311, 1990.
HINGSTON, F.J.; POSNER, A.M. \& QUIRK, J.P. Anion adsorption by goethite and gibbsite: I. The role of proton in determining adsorption envelopes. J. Soil Sci., 23:117192, 1972.

KEREN, R. \& BINGHAM, F.T. Boron in water, soils, and plants. Adv. Soil Sci., 1:229-276, 1985.

KEREN, R. \& MEZUMAN, U. Boron adsorption by clay minerals using a phenomenological equation. Clays Clay Miner., 29:198-204, 1981.

MARZADORI, C.; ANTISARI, L.V.; CIAVATTA, C. \& SEQUI, P. Soil organic matter influence on adsorption and desorption of boron. Soil Sci. Soc. Am. J., 55:1582-1585, 1991.

MEKARU, T. \& UEHARA, G. Anion adsorption in ferrugineous tropical soils. Soil Sci. Soc. Am. Proc., 36:296-300, 1972.

MEZUMAN, U. \& KEREN, R. Boron adsorption by soils using a phenomenological adsorption equation. Soil Sci. Soc. Am. J., 45:722-726, 1981.

NICHOLAICHUK, W.; LEYSHON, A.J.; JAME, Y.W. \& CAMPBELL, C.A. Boron and salinity survey of irrigation projects and the boron adsorption characteristics of some Saskatchewan soils. Can. J. Soil Sci., 68:77-90, 1988.

PRODROMOU, K.P. Boron adsorption by amorphous $\mathrm{Al}(\mathrm{OH})_{3}$ in the presence of low molecular weight organic acids. Agrochimica, 48:172-176, 2004.

SAS Institute. SAS user's guide: release 6.03. Cary, 1988.

SCHULTESS, C.P. \& DEY, D.K. Estimation of Langmuir constants using linear and nonlinear least square regression analysis. Soil Sci. Soc. Am. J., 60:433-442, 1996.

SIMS, J.R. \& BINGHAM, F.T. Retention of boron by layer silicates, sesquioxides and soil materials. II. Sesquioxides. Soil Sci. Soc. Am. Proc., 32:364-369, 1968.

SINGH, S.P.N. \& MATTIGOD, S.V. Modeling boron adsorption on kaolinite. Clays Clay Miner., 40:192-205, 1992.

SOARES, M.R.; ALLEONI, L.R.F. \& CASAGRANDE, J.C. Parâmetros termodinâmicos da reação de adsorção de boro em solos tropicais altamente intemperizados. Química Nova, 28:1014-1022, 2005.

VALLADARES, G.S.; PEREIRA, M.G. \& ALVES, G.C. Aplicação de duas isotermas de adsorção de boro em solos de baixada do Estado do Rio de Janeiro. R. Bras. Ci. Solo, 22:361-365, 1998

WEBER, O.L.S.; CHITOLINA, J.C.; CAMARGO, O.A. \& ALLEONI, L.R.F. Cargas elétricas estruturais e variáveis de solos tropicais altamente intemperizados. R. Bras. Ci. Solo, 29:867-873, 2005.

YERMIYAHU, U.; KEREN, R. \& CHEN, Y. Boron sorption by soil in the presence of composted organic matter. Soil Sci. Soc. Am. J., 59:405-409, 1995. 